\title{
Bound State Spectrum of Massive Thirring Model in Rest Frame
}

\author{
Makoto HIRAMOTO* and Takehisa FUJITA ${ }^{\dagger}$ \\ Department of Physics, Faculty of Science and Technology Nihon University, Tokyo, Japan
}

(November 23, 2018)

\begin{abstract}
The bound state spectrum of the massive Thirring model is studied in the framework of the canonical quantization in the rest frame. First, we quantize the field with the massless free fermion basis states. Then, we make a Bogoliubov transformation. This leads to the natural mass renormalization. The bound state spectrum is analytically solved by the $q \bar{q}$ Fock space. It is found that the spectrum has the right behaviors both for the weak and for the strong coupling limits after the appropriate wave function regularization. This regularization is quite clear and the treatment is self-consistent for the bound state problem compared to other regularizations. Further, we show that the interaction between $q \bar{q}$ bosons is always repulsive and therefore there is no bound state in the four fermion $(q q \bar{q} \bar{q})$ Fock space. This confirms that there is only one bound state in the massive Thirring model.
\end{abstract}

PACS numbers: $11.10 . \mathrm{Kk}, 03.70 .+\mathrm{k}$

\section{INTRODUCTION}

The bound state spectrum of the massive Thirring model has been studied by several methods. Unfortunately, several different methods give different results on the spectrum of the bound state. Dashen et al. investigated the bound state mass of the Sine-Gordon model. They applied the WKB method to the Sine-Gordon model and obtained the soliton-antisoliton bound state mass [3]

$$
\mathcal{M}_{n}=2 \mathcal{M} \sin \left(\frac{n \gamma}{16}\right) \quad n=1,2, \cdots<\frac{8 \pi}{\gamma}
$$

with

$$
\gamma=\frac{\beta^{2}}{1-\frac{\beta^{2}}{8 \pi}}=\frac{8 \pi}{1+2 \frac{g^{\prime}}{\pi}}
$$

where $g^{\prime}$ is the coupling constant with Schwinger's regularization in the massive Thirring model [8]. In order to examine the spectrum of Eq. (1.1), they solved the Bethe-Salpeter equation both in the Sine-Gordon model and in the massive Thirring model, and obtained the bound state mass assuming that the Bethe-Salpeter equation is solved with the same regularization as Schwinger's one. This result corresponds to Eq. (1.1) if we expand Eq. (1.1) with $\gamma$ for $n=1$. Furthermore, they concluded that all the bound states with $n$ less than $8 \pi / \gamma$ are stable, although the bound state with $n \geq 3$ are imbedded in the continuum state of $n=1$ bosons.

On the other hand, Fujita and Ogura obtained the bound state mass of the massive Thirring model employing the infinite momentum frame technique $(1 / K$ method) $[4$. There is only one bound state in this method. They presented the boson mass $\mathcal{M}$ (the fermion-antifermion bound state) as,

$$
\mathcal{M}=2 m \sin \theta,
$$

where $\theta$ is between 0 and $\pi / 2$ and is determined by

$$
\frac{1}{\theta \tan \theta}=\frac{g}{\pi}\left[1+\frac{1}{\sin ^{2} \theta}\left(1-\frac{g}{4 \pi}\right)\right],
$$

*e-mail: hiramoto@phys.cst.nihon-u.ac.jp

${ }^{\dagger}$ e-mail: fffujita@phys.cst.nihon-u.ac.jp 
where the coupling constant $g$ is Johnson's normalization [9]. Further, Fujita et al. solved the Bethe ansatz equation of the massive Thirring model numerically [5,6]. They confirmed that there is only one bound state. They also investigated the boson-boson scattering states in two-particle two-hole configurations. Further, it is found that all the rapidity variables are real. Therefore, they claimed that there is no string solution which satisfies the Bethe ansatz equations in two-particle two-hole states.

Recent calculations of the bound state spectrum for the massive Thirring model have shown that several different methods give different results on the normalization of the coupling constant [10]. This comes mainly from the normalization ambiguity of the coupling constant due to the fermion current regularization in the massive Thirring model. For the massless Thirring model, Klaiber proved that the coupling constant has a normalization ambiguity which arises from the fermion current regularization [11]. In the case of the massive Thirring model, it is expected that the same type of the coupling constant ambiguity may appear.

In this paper, we calculate the bound state spectrum of the charge zero sector in the massive Thirring model in the rest frame which is an opposite limit to the $1 / K$ method. The eigenvalue equation with the $q \bar{q}$ Fock space is solved analytically. It is shown that the spectrum has the same behaviors both for the weak and for the strong coupling limits as those of the other methods, but there still have some differences between the present calculation and other calculations due to the current regularization. In the case of the bound state problem, the regularization procedure is highly non trivial, since we have to sum up some types of the diagrams nonperturbatively in order to obtain the bound state. Here, we propose somewhat a different way to proceed the regularization. We carry out a regularization at the level of the bound state wave function in a consistent fashion. Further, in order to examine the number of bound states in the massive Thirring model, we consider the interaction between bosons in the four-fermion Fock space $(q q \bar{q} \bar{q})$. We check analytically that the interaction between $q \bar{q}$ bosons is always repulsive in the $q q \bar{q} \bar{q}$ Fock space and find that there is no excited bound state.

This paper is organized as follows. In the next section, we briefly explain the quantization of the massive Thirring model. Here, we quantize the fermion field with the free massless fermion basis states. In this basis states, the fermion mass is naturally renormalized after we make the Bogoliubov transformation. In Section 3, we obtain the eigenvalue equation in the $q \bar{q}$ Fock space. In Section 4, we present a regularization scheme of the bound state wave function. We also discuss some properties of the bound state spectrum of this equation. In section 5 , we evaluate the interaction between $q \bar{q}$ bosons of the massive Thirring model in the four-fermion Fock space $(q q \bar{q} \bar{q})$. It is found that the four-fermion Fock space does not produce any bound state. Finally, we summarize in Section 6 what we have clarified from the present paper.

\section{THE HAMILTONIAN OF THE MASSIVE THIRRING MODEL}

The massive Thirring model is a $(1+1)$ dimensional field theory which is described by the following Lagrangian density,

$$
\mathcal{L}=\bar{\psi}\left(i \gamma^{\mu} \partial_{\mu}-m_{0}\right) \psi-\frac{1}{2} g j_{\mu} j^{\mu}
$$

The fermion current $j^{\mu}$ is defined by

$$
j^{\mu}=: \bar{\psi} \gamma^{\mu} \psi:
$$

where we choose $\gamma$ matrices as

$$
\gamma^{0}=\left(\begin{array}{ll}
0 & 1 \\
1 & 0
\end{array}\right), \quad \gamma^{1}=\left(\begin{array}{cc}
0 & -1 \\
1 & 0
\end{array}\right), \quad \gamma^{5}=\left(\begin{array}{cc}
1 & 0 \\
0 & -1
\end{array}\right) .
$$

In this representation, the Hamiltonian is given as

$$
H=\int d x\left[-i \psi_{1}^{\dagger} \frac{\partial}{\partial x} \psi_{1}+i \psi_{2}^{\dagger} \frac{\partial}{\partial x} \psi_{2}+m_{0}\left(\psi_{1}^{\dagger} \psi_{2}+\psi_{2}^{\dagger} \psi_{1}\right)+2 g \psi_{1}^{\dagger} \psi_{2}^{\dagger} \psi_{2} \psi_{1}\right] .
$$

Now, we quantize the fermion field in a box $L$

$$
\begin{array}{r}
\psi(x)=\frac{1}{\sqrt{L}} \sum_{n}\left(\begin{array}{c}
a_{n} \\
b_{n}
\end{array}\right) e^{i p_{n} x}, \\
\left\{a_{i}, a_{j}^{\dagger}\right\}=\left\{b_{i}, b_{j}^{\dagger}\right\}=\delta_{i, j} .
\end{array}
$$


Then, the Hamiltonian becomes

$$
H=\sum_{n}\left[p_{n}\left(a_{n}^{\dagger} a_{n}-b_{n}^{\dagger} b_{n}\right)+m_{0}\left(a_{n}^{\dagger} b_{n}+b_{n}^{\dagger} a_{n}\right)+\frac{2 g}{L} \tilde{j}_{1, p_{n}} \tilde{j}_{2,-p_{n}}\right]
$$

where currents in the momentum representation $\tilde{j}_{1, p_{n}}$ and $\tilde{j}_{2, p_{n}}$ are given by

$$
\begin{aligned}
& \tilde{j}_{1, p_{n}}=\sum_{l} a_{l}^{\dagger} a_{l+n} \\
& \tilde{j}_{2, p_{n}}=\sum_{l} b_{l}^{\dagger} b_{l+n} .
\end{aligned}
$$

For the free field theory $(g=0)$, the Hamiltonian can be exactly diagonalized by a Bogoliubov transformation which mixes left- and right-handed fermions. On the other hand, this is not possible for interacting cases. However, for the massive Thirring model, we can get reliable results as we consider the bound state spectrum. Now, we introduce new fermion operators by a Bogoliubov transformation,

$$
\begin{aligned}
& \frac{c_{n}-d_{-n}^{\dagger}}{\sqrt{2}}=\cos \frac{\theta_{n}}{2} a_{n}+\sin \frac{\theta_{n}}{2} b_{n}, \\
& \frac{c_{n}+d_{-n}^{\dagger}}{\sqrt{2}}=-\sin \frac{\theta_{n}}{2} a_{n}+\cos \frac{\theta_{n}}{2} b_{n} .
\end{aligned}
$$

First, we consider the free field theory. In this case, the free field Hamiltonian $H_{0}$ can be written in terms of the new operators $c_{n}$ and $d_{n}$

$$
\begin{aligned}
H_{0}= & \sum_{n}\left[\left(p_{n} \sin \theta_{n}+m_{0} \cos \theta_{n}\right)\left(c_{n}^{\dagger} c_{n}+d_{-n}^{\dagger} d_{-n}\right)\right. \\
& \left.+\left(-p_{n} \cos \theta_{n}+m_{0} \sin \theta_{n}\right)\left(c_{n}^{\dagger} d_{-n}^{\dagger}+d_{-n} c_{n}\right)\right]
\end{aligned}
$$

The condition that the terms proportional to $\left(c^{\dagger} d^{\dagger}+c d\right)$ must vanish determines the theta parameter $\theta_{n}$. This is given as

$$
\tan \theta_{n}=\frac{p_{n}}{m_{0}}
$$

Then, the new free field Hamiltonian $H_{0}^{\prime}$ becomes

$$
H_{0}^{\prime}=\sum_{n} \sqrt{p_{n}^{2}+m_{0}^{2}}\left(c_{n}^{\dagger} c_{n}+d_{n}^{\dagger} d_{n}\right)
$$

Next, we make a Bogoliubov transformation Eq. (2.10) and (2.11) for the full Hamiltonian Eq. (2.7). Then, the new Hamiltonian $H^{\prime}$ becomes

$$
H^{\prime}=\tilde{H}_{0}+H_{c d}+H_{C}+H_{A}+H_{R}+H_{n o n}
$$

where $\tilde{H}_{0}$ denotes the free field Hamiltonian,

$$
\tilde{H}_{0}=\sum_{n}\left[\left\{p_{n} \sin \theta_{n}+\left(m_{0}+\frac{g}{L} \mathcal{A}\right) \cos \theta_{n}\right\}\left(c_{n}^{\dagger} c_{n}+d_{-n}^{\dagger} d_{-n}\right)\right],
$$

where $\mathcal{A}=\sum \cos \theta$ is related to the mass renormalization. We rewrite the fermion mass in terms of the renormalized mass $m$

$$
m=m_{0}+\frac{g}{L} \mathcal{A}
$$

Therefore, we can naturally renormalize the fermion mass.

The interacting Hamiltonian can be written as 


$$
\begin{aligned}
H_{C}=-\frac{2 g}{L} \sum_{k, p, q}\left\{\sin \frac{\theta_{p}}{2} \sin \frac{\theta_{p+k}}{2} \sin \frac{\theta_{q}}{2} \sin \frac{\theta_{q-k}}{2} c_{p}^{\dagger} c_{p+k} d_{-q+k}^{\dagger} d_{-q}\right. \\
\left.\quad+\cos \frac{\theta_{q}}{2} \cos \frac{\theta_{q-k}}{2} \cos \frac{\theta_{p}}{2} \cos \frac{\theta_{p+k}}{2} c_{q}^{\dagger} c_{q-k} d_{-p-k}^{\dagger} d_{-p}\right\}, \\
H_{A}=-\frac{2 g}{L} \sum_{k, p, q}\left\{\sin \frac{\theta_{p}}{2} \cos \frac{\theta_{q-k}}{2} \cos \frac{\theta_{p+k}}{2} \sin \frac{\theta_{q}}{2} c_{p}^{\dagger} c_{q-k} d_{-p-k}^{\dagger} d_{-q}\right. \\
\left.\quad+\cos \frac{\theta_{q}}{2} \sin \frac{\theta_{p+k}}{2} \sin \frac{\theta_{q-k}}{2} \cos \frac{\theta_{p}}{2} c_{q}^{\dagger} c_{p+k} d_{-q+k}^{\dagger} d_{-p}\right\}, \\
H_{R}=-\frac{2 g}{L} \sum_{k, p, q}\left\{\sin \frac{\theta_{p}}{2} \cos \frac{\theta_{q}}{2} \sin \frac{\theta_{p+k}}{2} \cos \frac{\theta_{q-k}}{2} c_{p}^{\dagger} c_{q}^{\dagger} c_{p+k} c_{q-k}\right. \\
\left.\quad+\cos \frac{\theta_{p}}{2} \sin \frac{\theta_{q}}{2} \cos \frac{\theta_{p+k}}{2} \sin \frac{\theta_{q-k}}{2} d_{-p-k}^{\dagger} d_{-q+k}^{\dagger} d_{-p} d_{-q}\right\},
\end{aligned}
$$

and $H_{\text {non }}$ denotes the term which does not conserve fermion antifermion number. The interacting Hamiltonian $H_{c d}$ describes the $\left(c^{\dagger} d^{\dagger}+c d\right)$ terms

$$
H_{c d}=\sum_{n}\left(-p_{n} \cos \theta_{n}+m \sin \theta_{n}\right)\left(c_{n}^{\dagger} d_{-n}^{\dagger}+d_{-n} c_{n}\right)
$$

It is interesting to note that the Hamiltonian $H_{c d}$ has the same shape as the free field Hamiltonian $H_{0}$. The important point is that the free field Hamiltonian has the bare mass $m_{0}$ after the Bogoliubov transformation while we have the renormalized mass in Eq. (2.21). We impose the same condition as the free field Hamiltonian. Thus, we obtain

$$
\tan \theta_{n}=\frac{p_{n}}{m}
$$

which is just the condition for the free field Hamiltonian except $m$. Then, $\tilde{H}_{0}$ is modified as

$$
\tilde{H}_{0}=\sum_{n} E_{p_{n}}\left(c_{n}^{\dagger} c_{n}+d_{n}^{\dagger} d_{n}\right)
$$

where $E_{p_{n}}=\sqrt{p_{n}^{2}+m^{2}}$.

This is all that is necessary to evaluate the bound state spectrum of the massive Thirring model.

\section{BOUND STATE SPECTRUM WITHIN $Q \bar{Q}$ FOCK SPACE}

In order to obtain physical quantities, we have to diagonalize the Hamiltonian. In this case, we have to prepare basis states. Here, we employ the Fock space expansion. We limit the Fock space to $q \bar{q}$ only, since there is no particle creation in the massive Thirring model, and therefore there is no mixture between $q \bar{q}$ and $q q \bar{q} \bar{q}$ Fock spaces.

Now, the Fock space for the $q \bar{q}$ state can be written

$$
|q \bar{q}\rangle=\sum_{n} f_{n} c_{n}^{\dagger} d_{-n}^{\dagger}|0\rangle
$$

where $f_{n}$ is a wave function in momentum space and satisfies the normalization condition, $\sum\left|f_{n}\right|^{2}=1$. The energy eigenvalue of the Hamiltonian with the $q \bar{q}$ Fock space can be written as

$$
\mathcal{M}=2 \sum_{n}\left|f_{n}\right|^{2} E_{p_{n}}-\frac{g}{L} \sum_{l, n} f_{l}^{\dagger} f_{n}\left(1+\sin \theta_{l} \sin \theta_{n}+\cos \theta_{l} \cos \theta_{n}\right) .
$$

Equivalently, we can write the equation by making variations with respect to $f_{n}$,

$$
\begin{aligned}
\mathcal{M} f_{n} & =2 E_{p_{n}} f_{n}-\frac{g}{L} \sum_{l} f_{l}\left(1+\sin \theta_{l} \sin \theta_{n}+\cos \theta_{l} \cos \theta_{n}\right), \\
& =2 E_{p_{n}} f_{n}-\frac{g}{L} \sum_{l} f_{l}\left(1+\frac{m^{2}}{E_{p_{n}} E_{p_{l}}}+\frac{p_{n} p_{l}}{E_{p_{n}} E_{p_{l}}}\right) .
\end{aligned}
$$


Converting the sum to the integral, we finally obtain

$$
\mathcal{M} f(p)=2 E_{p} f(p)-\frac{g}{2 \pi} \int d q f(q)\left(1+\frac{m^{2}}{E_{p} E_{q}}+\frac{p q}{E_{p} E_{q}}\right) .
$$

This equation is solved analytically, because it is a separable type interaction.

Now, we solve the integral equation (3.5). Here, we assume that the wave function satisfies the symmetric condition $f(-p)=f(p)$. In this case, we can drop the last term of Eq. (3.5). We define the following quantities $A$ and $B$

$$
\begin{aligned}
& A=\int_{-\infty}^{\infty} d p f(p), \\
& B=\int_{-\infty}^{\infty} d p \frac{f(p)}{E_{p}} .
\end{aligned}
$$

Using $A$ and $B$, we can solve Eq. (3.5) for $f(p)$ and obtain

$$
f(p)=\frac{g / 2 \pi}{2 E_{p}-\mathcal{M}}\left(A+\frac{m^{2}}{E_{p}} B\right)
$$

Putting this $f(p)$ back into Eqs. (3.6) and (3.7), we obtain the matrix equations

$$
\begin{aligned}
& A=\frac{g}{2 \pi} \int_{0}^{\Lambda} \frac{2 d p}{2 E_{p}-\mathcal{M}}\left(A+\frac{m^{2}}{E_{p}} B\right), \\
& B=\frac{g}{2 \pi} \int_{0}^{\infty} \frac{2 d p}{\left(2 E_{p}-\mathcal{M}\right) E_{p}}\left(A+\frac{m^{2}}{E_{p}} B\right) .
\end{aligned}
$$

Now, the regularization is necessary for the first equation, since the integral has the divergent term $\log [\Lambda / m+$ $\sqrt{1+(\Lambda / m)^{2}}$. This arises from the current-current interaction at the same space-time point. This must be regularized. In the next section, we treat the wave function regularization procedure.

\section{WAVE FUNCTION REGULARIZATION}

The regularization procedure is well defined for the perturbative treatment. There is no serious problem to renormalize divergent terms in the massive Thirring model. However, if we treat bound state problems, then the regularization procedure is highly nontrivial. This is because we have to sum up some of the diagrams nonperturbatively in order to obtain any bound states. For example, Dashen et al. [3] carried out the regularization of the coupling constant perturbatively for the bound state. There, they regularize the divergent term by first solving the eigenvalue equation and then by adding a new counter term to cancel the divergent part in the energy eigenvalue.

Here, we propose somewhat a different way to proceed the regularization. We carry out the regularization at the level of wave function in a consistent fashion. The origin of the divergent term comes from the nature of the current-current interaction $j_{\mu}(x) j^{\mu}(y)$ which has the divergent at the same space-time point $x=y$.

In our case, the divergent term $\log \left[\Lambda / m+\sqrt{1+(\Lambda / m)^{2}}\right]$ arises from the integration of $E_{p}^{-1}$. We subtract $g A / 4 \pi E_{p}$ from $f(p)$,

$$
\bar{f}(p)=f(p)-\frac{g}{2 \pi} \frac{A}{2 E_{p}} .
$$

In this case, we obtain

$$
\bar{f}(p)=\frac{g / 2 \pi}{\left(2 E_{p}-\mathcal{M}\right) E_{p}}\left(\frac{\mathcal{M}}{2} \bar{A}+m^{2} \bar{B}\right) .
$$

With this $\bar{f}(p)$, we obtain new matrix equations as

$$
\begin{aligned}
& \bar{A}=\frac{g}{2 \pi} \alpha^{-1}\left(\frac{\pi}{2}+\tan ^{-1} \frac{\mathcal{M}}{2 \alpha}\right)\left(\frac{\mathcal{M}}{2} \bar{A}+m^{2} \bar{B}\right), \\
& \bar{B}=\frac{g}{2 \pi} \frac{2}{\mathcal{M}}\left[\alpha^{-1}\left(\frac{\pi}{2}+\tan ^{-1} \frac{\mathcal{M}}{2 \alpha}\right)-\frac{\pi}{2 m}\right]\left(\frac{\mathcal{M}}{2} \bar{A}+m^{2} \bar{B}\right),
\end{aligned}
$$


where, $\alpha=\sqrt{m^{2}-(\mathcal{M} / 2)^{2}}$. Here, the divergent term is cancelled out. Therefore, the above equations are written by the finite quantities only. The divergent quantities are removed in a very nice and consistent fashion. We note that there are other regularizations for the current-current interaction at the same space-time point. One good example of the regularization could be $e^{i p \varepsilon}(\varepsilon \rightarrow 0)$. However, if one wants to apply $e^{i p \varepsilon}$ regularization to the bound state problem, one cannot regularize the wave function in a consistent way.

Now, we obtain the eigenvalue equation,

$$
\frac{g}{2 \pi}\left[\left(\frac{\mathcal{M}}{2 \alpha}+\frac{2 m^{2}}{\mathcal{M} \alpha}\right)\left(\frac{\pi}{2}+\tan ^{-1} \frac{\mathcal{M}}{2 \alpha}\right)-\frac{\pi m}{\mathcal{M}}\right]=1
$$

Introducing a new variable $\theta$ by

$$
\mathcal{M}=2 m \sin \theta
$$

Eq. (4.5) becomes

$$
\frac{g}{2 \pi}\left[\left(\tan \theta+\frac{1}{\sin \theta \cos \theta}\right)\left(\frac{\pi}{2}+\theta\right)-\frac{\pi}{2 \sin \theta}\right]=1,
$$

where $\theta$ is between 0 and $\pi / 2$.

Now, we check the strong coupling limit $\theta \sim 0$. This corresponds to $\mathcal{M}=2 m \sin \theta \sim 2 m \theta$. From Eq. (4.7), we get

$$
\theta \sim \frac{2}{3 \pi}\left(2-\frac{g}{\pi}\right)
$$

Therefore, the bound state spectrum becomes

$$
\mathcal{M} \sim \frac{4}{3 \pi}\left(2-\frac{g}{\pi}\right) m
$$

Namely, $\mathcal{M}$ becomes zero for $g / \pi=2$. Beyond that, there is no bound state and the theory is not well defined. This is precisely what is predicted by Johnson [9]. This is because we use the massless free fermion basis states. In this case, the coupling constant normalization is bound to use Johnson's normalization as Klaiber shows [11. This situation can be seen easily in terms of bosonized form of the Thirring model. Since the free fermion current satisfies boson's commutation relations, the bosonized Hamiltonian of the Thirring model can be written as

$$
H=\sum_{p}\left[\left(1-\frac{g}{2 \pi}\right) \Pi^{\dagger}(p) \Pi(p)+\left(1+\frac{g}{2 \pi}\right) p^{2} \Phi^{\dagger}(p) \Phi(p)\right],
$$

where $\Phi(p)$ and $\Pi(p)$ are related to the free fermion current as

$$
\begin{aligned}
& \tilde{J}_{0}(p)=\tilde{j}_{1, p}+\tilde{j}_{2, p}=i p \sqrt{\frac{L}{\pi}} \Phi(p) \\
& \tilde{J}_{0}(p)=\tilde{j}_{1, p}-\tilde{j}_{2, p}=-\sqrt{\frac{L}{\pi}} \Pi(p) .
\end{aligned}
$$

Therefore, we can see that

$$
-2 \leq \frac{g}{\pi} \leq 2
$$

which is just Johnson's constraint. For the massive Thirring model, we should add the mass term $m \bar{\psi} \psi$ to the Lagrangian. However, the mass term can be described only by $\Phi(p)$ without kinetic terms, and therefore it does not influence the condition that $g / \pi$ should be smaller than 2 .

Next, we consider the weak coupling limit $(g / \pi \sim 0)$. In this case, we put $\theta=\pi / 2-\beta(\beta \sim 0)$. Then, we obtain the bound state spectrum

$$
\mathcal{M}=2 m \cos \beta=m\left[2-g^{2}+\left(2+\frac{\pi}{2}\right) \frac{g^{3}}{\pi}+O\left(g^{4}\right)\right] .
$$

This result can be compared with the prediction of Fujita and Ogura in the $1 / K$ method calculation 


$$
\mathcal{M}=m\left[2-g^{2}+\left(4+\frac{1}{4}\right) \frac{g^{3}}{\pi}+O\left(g^{4}\right)\right] .
$$

On the other hand, the WKB formula Eq. (1.1) with Johnson's regularization becomes

$$
\mathcal{M}=m\left[2-g^{2}+3 \frac{g^{3}}{\pi}+O\left(g^{4}\right)\right] .
$$

As can be seen, the coefficients of $g^{3}$ are different from each other. This suggests that each method has nice features both for the weak and for the strong coupling limits, but there still have ambiguities which are related to the currentcurrent regularization.

In Fig. 1, we show numerical results of Eq. (4.7), the $1 / K$ method, the WKB method and the Bethe ansatz solution. As can be seen from Fig. 1, the difference between the present calculation and the WKB method is surprisingly small, while the difference between the present calculation and the $1 / K$ method is not so small. This suggests that the regularization may well be related to the total momenta $K$ of $q \bar{q}$.

\section{FOUR-FERMION FOCK SPACE}

In this section, we consider the four-fermion Fock space. To include the four-fermion Fock space, we must be careful for treating transitions from $q \bar{q}$ to $q q \bar{q} \bar{q}$ Fock space. It is well known that the transition that changes fermion numbers is forbidden in the massive Thirring model. This is due to an infinite number of conservation laws that must be obeyed by the external momenta [14, 15]. Here, we consider the $H_{R}$ term. This contributes to the higher order Fock space

Now, we calculate the expectation value of $H_{R}$ in the $q q \bar{q} \bar{q}$ Fock space. The $q q \bar{q} \bar{q}$ Fock space is defined by

$$
|q q \bar{q} \bar{q}\rangle=\sum_{p_{1} p_{2} q_{1} q_{2}} f^{(4)}\left(p_{1}, p_{2} ; q_{1}, q_{2}\right) c_{p_{1}}^{\dagger} c_{p_{2}}^{\dagger} d_{q_{1}}^{\dagger} d_{q_{2}}^{\dagger}|0\rangle \delta_{p_{1}+p_{2}+q_{1}+q_{2}, 0}
$$

where $f^{(4)}\left(p_{1}, p_{2} ; q_{1}, q_{2}\right)$ has an antisymmetric property;

$$
f^{(4)}\left(p_{2}, p_{1} ; q_{1}, q_{2}\right)=f^{(4)}\left(p_{1}, p_{2} ; q_{2}, q_{1}\right)=-f^{(4)}\left(p_{1}, p_{2} ; q_{1}, q_{2}\right) .
$$

Thus, we obtain

$$
\begin{aligned}
\mathcal{E}_{\mathcal{R}} & =\left\langle q q \bar{q} \bar{q}\left|H_{R}\right| q q \bar{q} \bar{q}\right\rangle \\
& =\frac{8 g}{L} \sum_{p_{1} p_{2} q_{1} q_{2}}\left|f^{(4)}\left(p_{1}, p_{2} ; q_{1}, q_{2}\right)\right|^{2}\left(\sin \theta_{p_{1}} \cos \theta_{p_{2}}-\cos \theta_{p_{1}} \sin \theta_{p_{2}}\right)^{2} .
\end{aligned}
$$

Now, we see that $\mathcal{E}_{R}$ is always positive. Therefore, this is repulsive and thus there is no bound state in the four fermion Fock space. From this calculation, we conclude that there is only one bound state in the massive Thirring model.

\section{CONCLUSIONS}

We have presented the bound state spectrum in the massive Thirring model. This is based on the $q \bar{q}$ Fock space in the rest frame. The eigenvalue equation with the $q \bar{q}$ Fock space is solved analytically. It is shown that the bound state spectrum has right behaviors both for the weak and for the strong coupling limits after the appropriate wave function regularization. This is indeed confirmed since the $q \bar{q}$ Fock space has no transition to higher Fock space due to an infinite number of conservation law. Further, in order to confirm that there is only one bound state, we carry out the analytic evaluation of the interaction term in the $q q \bar{q} \bar{q}$ Fock space. We show that the interaction between the bosons of $q \bar{q}$ Fock space is repulsive and therefore there is no bound state in the four-fermion Fock space.

Although there are still some differences between the present result and other methods, we can reliably calculate the bound state spectrum after the appropriate wave function regularization. At the present stage, it is not so clear whether the regularization ambiguity of the massive Thirring model can be different from the massless Thirring model. It may well be that the regularization ambiguity is related to some hidden symmetry which is not yet clearly understood up to now. 


\section{ACKNOWLEDGMENTS}

We thank K. Yazaki for careful reading of the manuscript and for stimulating discussions.

[1] H. Bergknoff and H. B. Thacker, Phys. Rev. Lett. 42 (1979) 135.

[2] H. B. Thacker, Rev. Mod. Phys. 53 (1981) 253.

[3] R. F. Dashen, B. Hasslacher and A. Neveu, Phys. Rev. D11 (1975) 3424.

[4] T. Fujita and A. Ogura, Prog. Theor. Phys. 89 (1993) 23.

[5] T. Fujita, Y. Sekiguchi and K. Yamamoto, Ann. Phys. 255 (1997) 204.

[6] T. Fujita and M. Hiramoto, Phys. Rev. D58 (1998) 125019.

[7] M. Cavicchi, Int. J. Mod. Phys. A10 (1995) 167.

[8] J. Schwinger, Phys. Rev. Lett. 3 (1959) 296.

[9] K. Johnson, Nuovo Cimento 20 (1961) 773.

[10] T. Fujita, T. Kake and H. Takahashi, Ann. Phys. 282 (2000) 100.

[11] B. Kleiber, in Lectures in Theoretical Physics, 1967, edited by A. Barut and W. Britten (Gordon and Breach, 1968).

[12] N. Nakanishi, Prog. Theor. Phys. 57 (1997) 580.

[13] H. Takahashi and A. Ogura, Prog. Theor. Phys. 105 (2001) 495.

[14] B. Berg, M. Karowski, and H. -J. Thun, Phys. Lett. B62 (1976) 187.

[15] B. Berg, Nuovo Cimento 41A (1977) 58.

\section{FIGURE CAPTIONS}

FIG. 1. We show the bound state spectrum for the massive Thirring model with the $q \bar{q}$ Fock space as the function of $g / \pi$. Here, the coupling constant is Johnson's normalization. The solid line is calculated by Eq. (4.7) while the dotted line is calculated by Eq. (1.4). The dashed line is calculated by Eq. (1.1) with $n=1$. The dot-dashed line indicates the twice of Eq. (1) with $n=1$. We also plot the Bethe ansatz results with error bars. 


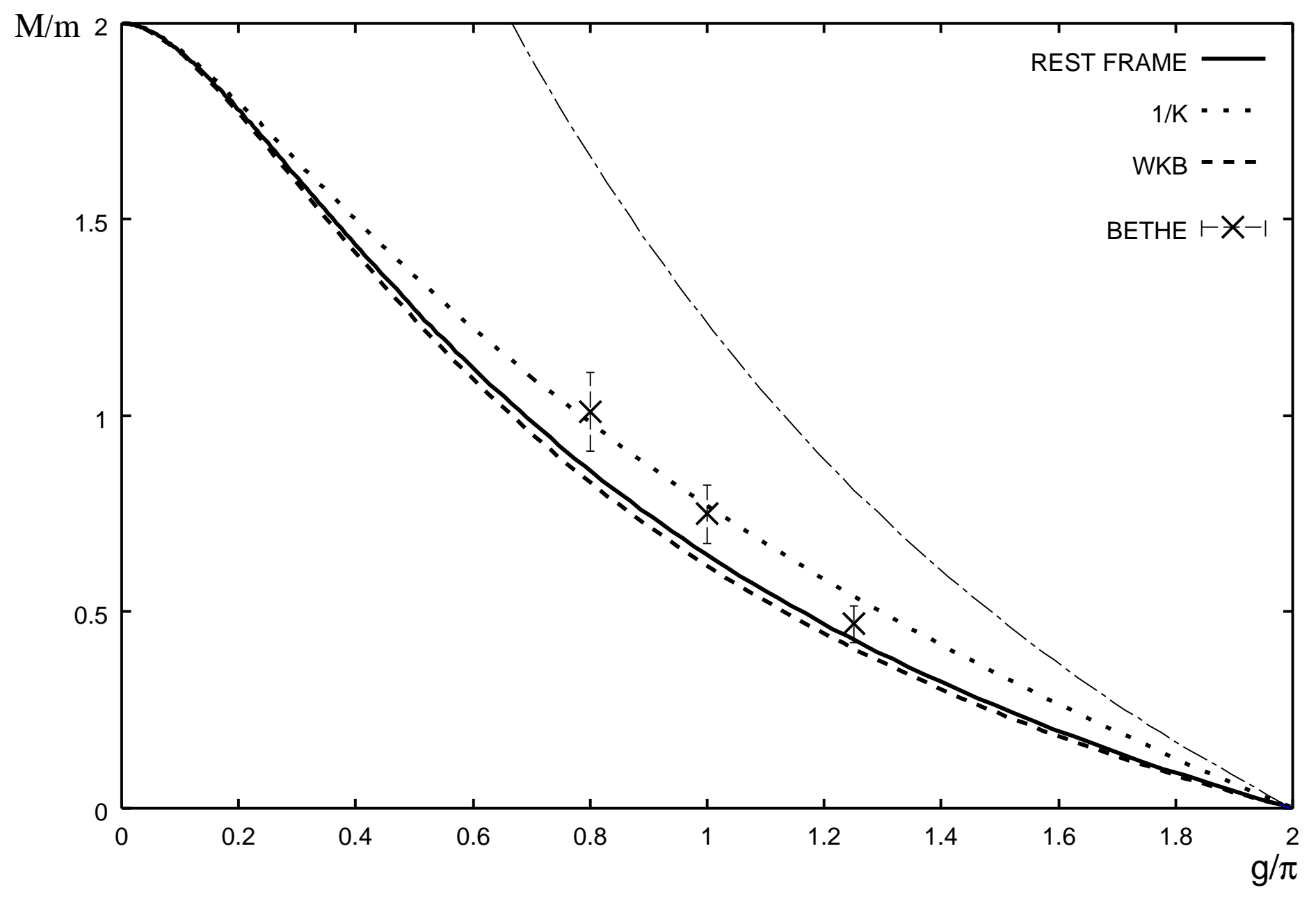

